\title{
A long-range polarization-controlled optical tractor beam
}

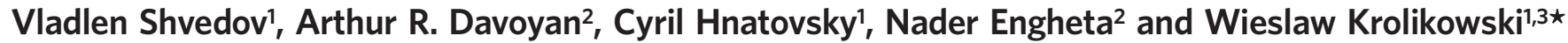

The laser beam has become an indispensable tool for the controllable manipulation and transport of microscopic objects in biology, physical chemistry and condensed matter physics. In particular, 'tractor' laser beams can draw matter towards a laser source and perform, for instance, all-optical remote sampling. Recent advances in lightwave technology have already led to small-scale experimental demonstrations of tractor beams ${ }^{1-4}$. However, the realization of long-range tractor beams has not gone beyond the realm of theoretical investigations $\mathrm{s}^{5-9}$. Here, we demonstrate the stable transfer of gold-coated hollow glass spheres against the power flow of a single inhomogeneously polarized laser beam over tens of centimetres. Additionally, by varying the polarization state of the beam we can stop the spheres or reverse the direction of their motion at will.

Traditional optical tweezers are based on the transfer of photon momenta to the particle to be manipulated ${ }^{10,11}$. The combined action of the scattering and gradient force, both of which originate from this effect, is often sufficient to trap and precisely move transparent micro-objects within a small region, typically limited to the focal volume of the tightly focused laser beam ${ }^{11,12}$. This type of optical manipulation is usually carried out in vacuum ${ }^{13}$ or, at another extreme, in liquids with a sufficiently high thermal conductivity ${ }^{14}$.

For light-absorbing particles immersed in a gaseous medium, the very possibility of using the momentum of light for optical tweezing is drastically reduced because even weak light absorption by the particles leads to the appearance of a strong gas-dynamic force acting upon them ${ }^{15,16}$. The latter light-induced force, known as the photophoretic force, provides at the same time an alternative and much more efficient way ${ }^{16}$ to control the motion of absorbing particles in gases within a broad range of pressures ${ }^{17,18}$.

When a photon is absorbed by a particle its momentum contributes to radiation pressure exerted on the particle, while its energy is converted into the thermal energy of the particle. The heated site on the particle surface then transfers this energy to the 'mediator', that is, the surrounding gas ${ }^{19}$. In the limiting case of thermally non-conductive particles placed in a highly rarefied gas, the force imparted by the gas molecules can be $\sim c / v$ times greater than the force originating from the transfer of photon momenta ${ }^{20}$, such as the scattering and gradient force ( $c$ is the speed of light and $v \ll c$ is the characteristic molecular velocity). This advantage, albeit to a lesser extent, exists in many other situations and, by relaxing the already mentioned highly restrictive conditions imposed on both the focusing geometry and the particle size, allows one to design photophoretic optical traps, inside which much larger objects can be stored, manipulated or moved over macroscopic distances ${ }^{21}$.

The photophoretic force $\mathbf{F}$, both in the slip-flow and free molecular regime, is collinear with the asymmetry vector $\mathbf{r}_{\mathrm{as}}$ given by
$\mathbf{r}_{\mathrm{as}}=C \int_{V_{\mathrm{p}}} q(\mathbf{r}) \mathbf{w}(\mathbf{r}) \mathrm{d} V$, where $C$ is a constant, $q(\mathbf{r})$ is the heat source distribution inside the particle originating from the absorption of light, $\mathbf{w}(\mathbf{r})$ is a vector weight function representing the solution of the pertinent boundary value problem for Laplace's equation inside the particle, $V_{\mathrm{p}}$ is the particle volume, and $\mathbf{r}$ is the position vector ${ }^{19,22-25}$. Generally, the photophoretic force can have any angle with respect to the light propagation direction, depending on the particle and light field parameters ${ }^{23}$.

The photophoretic force tends to eject absorbing particles from the regions of maximum light intensity ${ }^{26}$, thus precluding robust optical trapping and manipulation with beams that have a Gaussian intensity profile (Fig. 1a). The use of light fields that have distinct intensity minima where particles can be retained provides an elegant solution to this problem ${ }^{21,27}$. In principle, any doughnut beam with a phase or polarization singularity and zero light intensity along its axis ${ }^{21,28}$ satisfies the above criterion (Fig. 1b).

When an isotropic spherical particle is trapped inside such a beam and no external forces are acting on the particle, the photophoretic force is directed exactly parallel to the beam axis owing to the perfect axial symmetry of the problem. In this case the behaviour of the particle is determined by the asymmetry parameter $J$ (termed the $J$-factor hereon) ${ }^{19,22}$, which is the scalar version of $\mathbf{r}_{\text {as }}$. Similar to $\mathbf{r}_{\mathrm{as}}$, the $J$-factor represents a weighted integration of the heat source distribution over the particle volume.

A particle may absorb electromagnetic energy predominantly near the illuminated front side $(J<0)$, and the photophoretic force in this case pushes the particle along the beam propagation direction $^{21}$. On the other hand, when the energy is mainly absorbed inside the rear side of the particle, the particle may be pulled against the beam $(J>0)$. Satisfying the latter condition is, in fact, tantamount to creating the elusive 'tractor' beam, which can trap and simultaneously convey objects against the laser power flow. Here, we show that the sign of the $J$-factor and, as a consequence, the direction of the particle motion can be switched by adjusting the polarization state of an otherwise identical doughnut beam.

In our analysis we use semitransparent spheres (Fig. 1c) illuminated with either a radially or azimuthally polarized $\mathrm{TEM}_{01}{ }^{\star}$ beam, whose electric field in both the cases is linearly polarized at each point and can be written as $\mathbf{E}_{\rho}=E(\rho, z) \mathbf{e}_{\rho}$ and $\mathbf{E}_{\varphi}=E(\rho, z) \mathbf{e}_{\varphi}$, respectively (see Methods). In the paraxial approximation these beams have identical cross-sectional intensity distributions and can be converted into each other by straightforward polarization transformations ${ }^{28}$.

Figure 2a,b shows the light intensity distribution around and inside a Au-coated hollow glass sphere placed on the axis of a radially and azimuthally polarized beam, respectively. These intensity distributions determine the corresponding heat source

'Laser Physics Centre, Research School of Physical Sciences and Engineering, Australian National University, Canberra, Australia, ${ }^{2}$ Department of Electrical and Systems Engineering, University of Pennsylvania, Philadelphia, Pennsylvania 19104, USA, ${ }^{3}$ Science Program, Texas A\&M University at Qatar, Doha, Qatar. *e-mail: wzk111@physics.anu.edu.au 

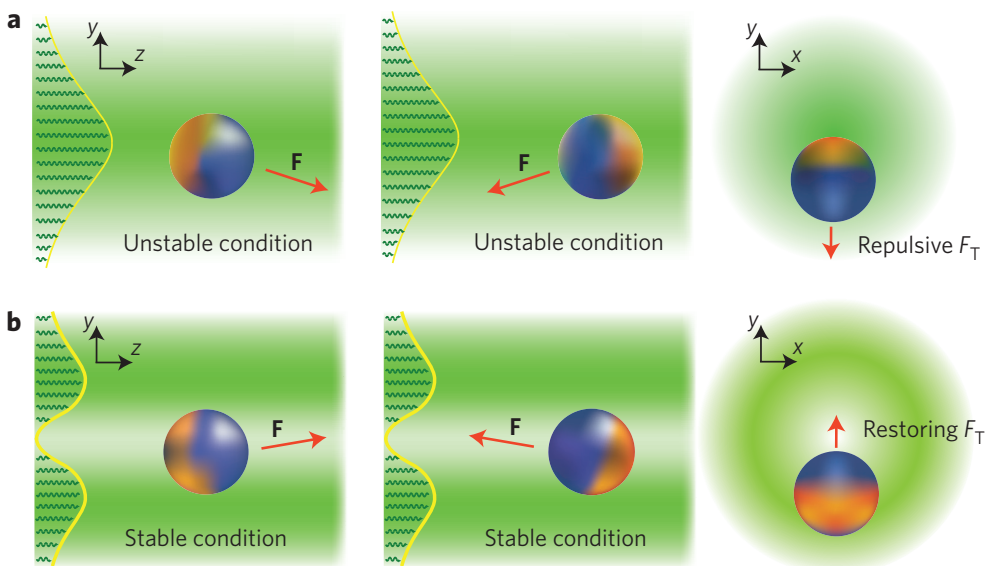

Figure 1 | Concept of photophoretic light-particle interaction in gases. a, Interaction of an absorbing particle with a Gaussian beam. The photophoretic force $\mathbf{F}$ can drive the particle along the beam in the forward or backward direction, depending on the asymmetry vector $\mathbf{r}_{\text {as. }}$. However, this motion is laterally unstable, as the transverse component of the photophoretic force $F_{\mathrm{T}}$ is repulsive and therefore the particle is pushed away from the maximum of light intensity centred at the beam axis. $\mathbf{b}$, Interaction of the same particle with a doughnut beam. In this case $F_{\mathrm{T}}$ is restoring (that is, it is directed towards the beam axis) and does not allow the particle to escape from the beam, so the motion is laterally stable. $\mathbf{c}$, A hollow glass sphere (external radius $a$, wall thickness $d$ ) coated with a thin Au film (film thickness $h$ ) exemplifies a semitransparent particle.
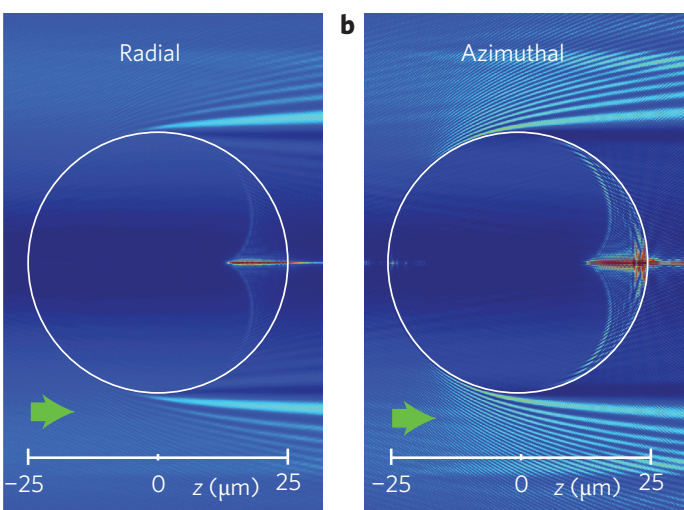

c

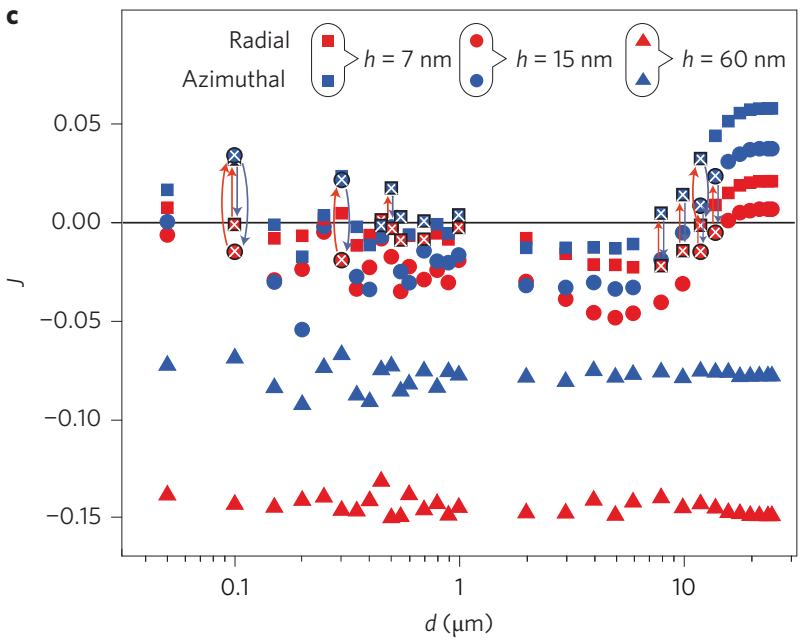

d

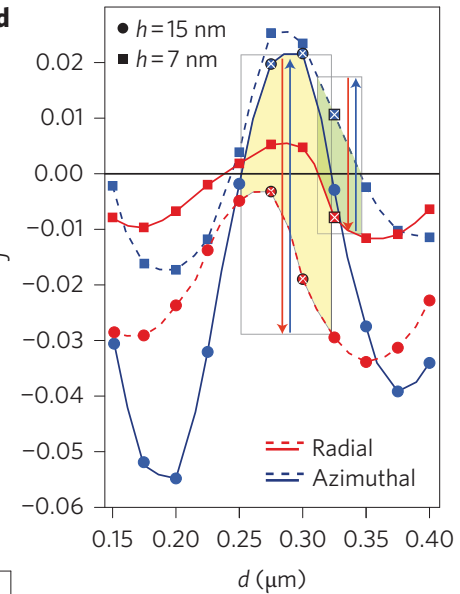

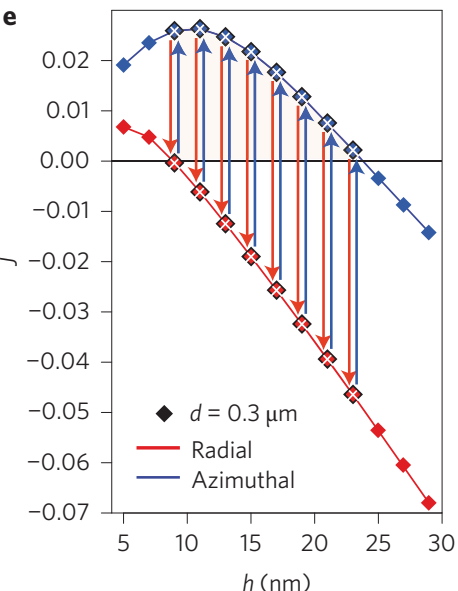

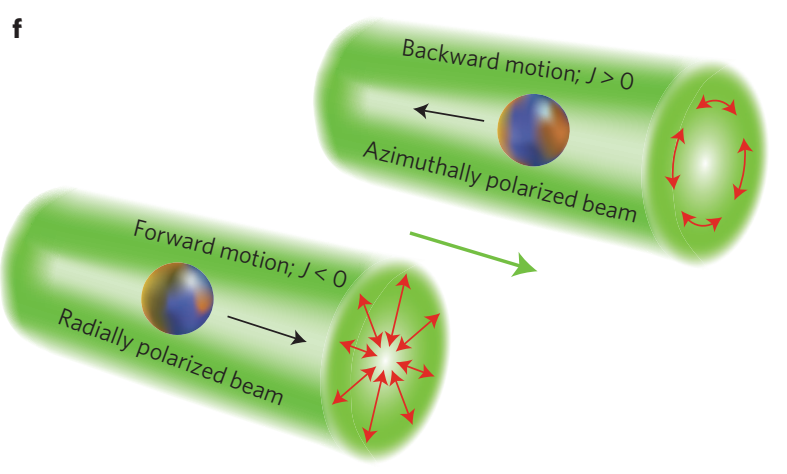

Figure 2 | Numerical modelling of reversible optical transport. a,b, Cross-sections of light intensity distribution near a partially absorbing hollow glass sphere $(d=300 \mathrm{~nm} ; h=10 \mathrm{~nm})$ trapped at the axis of a radially (a) and azimuthally (b) polarized beam. The distributions are axially symmetric with respect to the $z$-axis. c-e, Polarization-induced reversal of the $J$-factor of a hollow Au-coated glass sphere. In $\mathbf{c}$ and $\mathbf{e}$, the pairs of points connected with arrows indicate when either a pushing $(J<0)$ or pulling $(J>0)$ photophoretic force can be exerted on the sphere depending on the incident beam polarization. In $\mathbf{d}$, the shaded regions show when the reversible optical transport occurs for $7 \mathrm{~nm}$ (green) and $15 \mathrm{~nm}$ (yellow) Au coatings. In a-e the spheres have the same external radius $a \approx 25 \mu \mathrm{m}$, and the radius of the maximum intensity contour at the beam waist $(z=0)$ is $w_{0}=35 \mu \mathrm{m}$. $\mathbf{f}$, Illustration of polarization-controlled optical transport. 

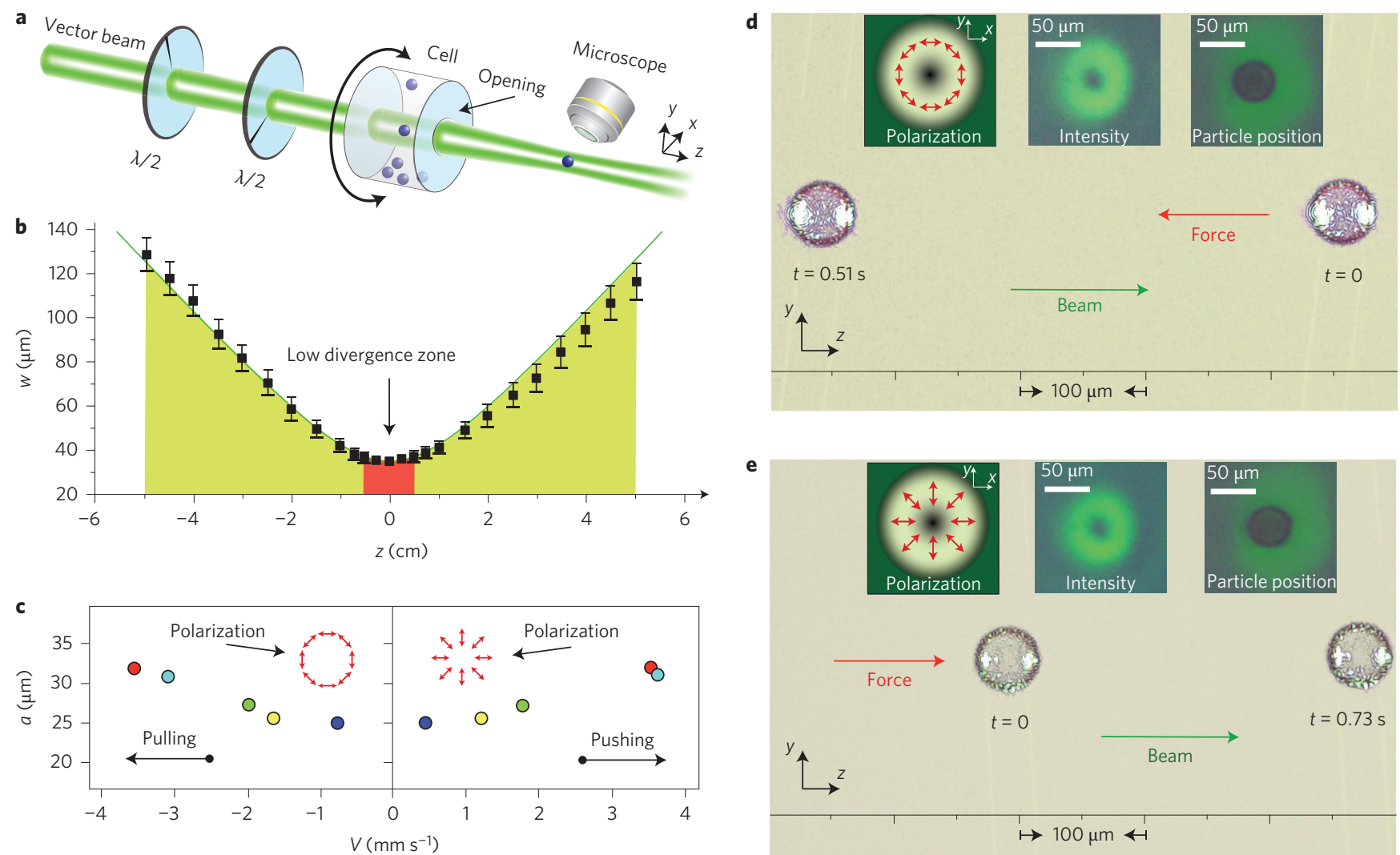

Figure 3 | Experiment. a, Part of the experimental set-up with the beam convertor and particle dispenser. The half-wave plates used to change the polarization state of the beam are denoted by $\lambda / 2$. $\mathbf{b}$, Illustration of the longitudinal light intensity profile of the trapping beam. Each black square represents measured light intensity, and the solid line is an analytical fit based on $w(z)=w_{0}\left(1+z^{2} \lambda_{0}^{2} /\left(4 \pi^{2} w_{0}^{4}\right)\right)^{1 / 2}$, where $w_{0}$ is the radius of the maximum intensity contour at the beam waist $(z=0)$. The region of stable trapping is in yellow. The beam waist region is in red. $\mathbf{c}$, Dependence of the velocity of the glass shells on their external diameter for azimuthal and radial polarization. Data pertaining to a particular shell are in one colour. In all the experiments $w_{0}=35 \mu \mathrm{m}$. d,e, Snapshots illustrating the motion of a shell of radius $a=25 \mu \mathrm{m}$ and coating thickness $h \approx 15 \mathrm{~nm}$ trapped inside an azimuthally (d) and radially (e) polarized beam. In $\mathbf{d}$, the shell moves against the beam propagation direction $z$ (pulling force) at $V=0.8 \mathrm{~mm} \mathrm{~s}^{-1}$, and in $\mathbf{e}$ it moves along $z$ (pushing force) at $V=0.4 \mathrm{~mm} \mathrm{~s}^{-1}$.

distributions $q(\mathbf{r})$ and $J$-factors ${ }^{19}$. When the coating is very thin (see Methods), the $J$-factor can be written as ${ }^{22}$

$$
J \approx 6 \pi a n \kappa \lambda_{0}^{-1} \int_{1-h / a}^{1} \int_{0}^{2 \pi}\left(|\mathbf{E}|^{2} /\left|\mathbf{E}_{\rho, \varphi}\right|^{2}\right) t^{3} \cos \theta \sin \theta \mathrm{d} \theta \mathrm{d} t
$$

where $\mathbf{E}$ is the electric field inside the light-absorbing coating, $n$ and $\kappa$ are respectively the real and imaginary parts of the refractive index of $\mathrm{Au}, a$ is the external radius of the sphere, $\lambda_{0}$ is the wavelength in vacuum, $t=r / a$, and $h$ is the coating thickness (Fig. 1c). The numerical analysis presented in Fig. 2c-e shows that, for a fixed external radius, both the magnitude and sign of the $J$-factor for either of the two polarization states critically depend on the glass wall and coating thickness. When the coating thickness is less than $\sim 25 \mathrm{~nm}$, the sphere can be regarded as semitransparent, implying that a significant portion of light is absorbed at its rear surface. As the wall thickness changes, the $J$-factor and, as a result, the photophoretic force itself, alternate due to the concomitant redistribution of absorbed energy over the sphere's surface. In fact, there are distinct wall thickness intervals where it is possible to change the sign of the photophoretic force exerted on the sphere and thus reverse the direction of its motion by solely switching the polarization state from radial to azimuthal. On the other hand, if the coating thickness exceeds $\sim 25 \mathrm{~nm}$, the $J$-factor is negative for any wall thickness and the corresponding photophoretic force becomes pushing, irrespective of the polarization state. For a $60 \mathrm{~nm}$ coating, for instance, the sphere is highly opaque and the incident light is mainly absorbed at its front surface. In this case, the beam polarization can only affect the magnitude of the $J$-factor ${ }^{28}$.

To demonstrate the predicted effects experimentally we used thin-walled spherical glass shells with $a$ varying from 25 to $35 \mu \mathrm{m}$ and $d$ from 200 to $400 \mathrm{~nm}$. The shells were sputtercoated with a thin layer of Au with $h$ varying from 7 to $15 \mathrm{~nm}$. For these parameters the sign of the photophoretic force is expected to be sensitive to the polarization state of the beam (Fig. 2d,e). To prepare the tractor beam we first converted a circularly polarized single-charge vortex beam into a superposition of radially and azimuthally polarized vector modes by means of a uniaxial birefringent crystal, and after that selected the azimuthal mode using a spatial filter ${ }^{28}$. When necessary, the thus synthesized azimuthally polarized beam was converted into a radially polarized one with two half-wave plates $(\lambda / 2$ in Fig. 3a), without affecting any other beam parameters ${ }^{28}$. The experiments were carried out using a $200 \mathrm{~mW}$ continuous-wave $532 \mathrm{~nm}$ laser beam with a fixed waist radius of $w_{0}=35 \mu \mathrm{m}$. These beam parameters allowed us to match the intensity distribution with the size of the shells.

To place the shells into the laser beam we used a cylindrical cuvette. On rotating the cuvette, some of the shells detached from its walls, fell, and were trapped inside the intensity minimum at the beam axis. In our experiments the beam axis was always perpendicular to the gravitational force acting on the shells. 
Openings at the end faces of the cuvette allowed the particles to escape from the cuvette and then migrate inside the beam over tens of centimetres in ambient air towards the beam waist (Fig. 3a). Generally, the photophoretic force changes along the beam axis due to beam divergence. With a view to reducing this effect, we measured the direction and magnitude of the shell velocity within $\mathrm{a} \sim 0.5 \mathrm{~mm}$ interval along the $z$-axis near the beam waist (region in red in Fig. 3b). Under these conditions, the beam cross-section changes by a mere $0.1 \%$ and the photophoretic force can therefore be assumed to remain constant. According to the charge-coupled device (CCD) images shown in Fig. 3d,e the shells were kept almost exactly at the beam axis in this region.

The motion of the shells along the beam axis was governed by the combined action of the photophoretic force and the Stokes frictional force $F_{\mathrm{S}}=6 \pi a \eta V$, where $\eta$ is the dynamic viscosity of air and $V$ is the shell velocity. Data analysis also showed that the shell velocity in the observation region was uniform to within $5 \%$, which allowed us to find the longitudinal component of the photophoretic force by equating it to $F_{\mathrm{S}}$. For instance, for the glass shell in Fig. 3d,e the pulling force is $\sim 6.7 \mathrm{pN}$ and the pushing force is $\sim 3.4 \mathrm{pN}$. Meanwhile, the transverse component of the photophoretic force $F_{\mathrm{T}}$ (Fig. 1a,b), which counterbalances the gravitational force acting on the shell, is $\sim 65 \mathrm{pN}$, based on a $\sim 6.5 \mathrm{ng}$ mass of the shell (see Methods).

Our key experimental data are summarized in Fig. 3c-e. First, an azimuthally polarized laser beam can become a tractor beam for certain types of weakly absorbing glass shell. Second, their direction of motion can be switched from backward to forward by changing the polarization state from azimuthal to radial. Conditions under which this becomes possible can still be predicted by employing the $J$-factor formalism. The presented tunable optical beam can transport absorbing particles in air over macroscopic distances (that is, tens of centimetres). In addition, the shells can be stopped at any position along the beam axis by setting the angle between the fast axes of the two half-wave plates somewhere between 0 and $\pi / 4$. In this case we generate a spirally polarized beam ${ }^{28}$.

Such long-distance, stable and switchable optical transport achieved with a single laser beam can find applications in various fields of science and technology, including laboratory-based studies of aerosols and remote sampling. Furthermore, the concept of polarization-controlled photophoretic transport is applicable to any gaseous media at pressures starting from $0.1 \mathrm{~Pa}$ and also to a wide class of liquids ${ }^{18,29}$. More complex scenarios of highprecision multidirectional manipulation and transport based on a network of beams with variable states of polarization may also be considered in the future.

\section{Methods}

The electric fields of a $\mathrm{TEM}_{01}{ }^{*}$ radially and azimuthally polarized beam in the cylindrical coordinate system (Fig. 1c) can be written as:

$$
\begin{aligned}
& \mathbf{E}_{\rho}=E(\rho, z) \mathbf{e}_{\rho}=E_{0}\left(\rho / w_{0} \xi^{2}\right) e^{-\rho^{2} / 2 w_{0}^{2} \xi} e^{i k z} \mathbf{e}_{\rho} \\
& \mathbf{E}_{\varphi}=E(\rho, z) \mathbf{e}_{\varphi}=E_{0}\left(\rho / w_{0} \xi^{2}\right) e^{-\rho^{2} / 2 w_{0}^{2} \xi} e^{i k z} \mathbf{e}_{\varphi}
\end{aligned}
$$

where $\mathbf{e}_{\rho}$ and $\mathbf{e}_{\varphi}$ denote the radial and azimuthal unit vectors, respectively, $\rho$ is the radial coordinate of an arbitrary point measured from the beam axis, $E_{0}$ is a normalization constant, $\xi(z)=1+i z \lambda_{0} /\left(2 \pi w_{0}^{2}\right)$ is a complex parameter characterizing the beam divergence, $w_{0}$ is the radius of the maximum intensity contour at the beam waist located at $z=0$, and $k=2 \pi / \lambda_{0}$.

The thermal conductivity of our metallized hollow spheres depends only on the radial coordinate $r$. According to Yalamov ${ }^{24}$, a spherical particle with a variable internal thermal conductivity described by $f(r)$ and a density of heat sources within it given by $q(r, \theta)$ can be regarded as a homogeneous particle with a constant thermal conductivity and an effective heat-source density $\mathbf{w}(r) q(r, \theta)$, where the weight function $\mathbf{w}(r)$ is determined by $f(r)$. Because the Au coating is very thin and the glass walls are totally transparent, the heat is released only at the particle surface where, by definition, $\mathbf{w}(r) \approx 1$. The intensity and polarization distribution of the doughnut beam that we consider here are axially symmetric. This removes the dependence of the source on the polar $\varphi$ coordinate and therefore allows us to write it as $q(r, \theta)$.

The numerical simulations were performed using the finite-element method commercially implemented in COMSOL Multiphysics. Because of the axial symmetry of the problem (that is, no variation of fields and forces with the $\varphi$ coordinate), the numerical analysis is significantly simplified (we used COMSOL's $2 \mathrm{D}$ axis symmetric solver). Due to the computationally demanding simulations, the data shown in Fig. 2e were obtained only for a discrete set of glass wall thicknesses (Fig. 2e). The permittivity of Au (and its refractive index) is deduced from the Drude-Lorentz model fitted to the experimental data ${ }^{30}$. The refractive index of the glass shells gives $n_{1} \approx 1.54$ and $\kappa_{1}=0$.

To calculate the longitudinal pulling and pushing force on a shell we first launched the shell into the radially polarized beam at $z \approx-50 \mathrm{~mm}$ from the beam waist. When the shell crossed the beam waist at $z \approx 0$, CCD images were taken at equal time intervals to record its position. When the shell was at $z \approx+50 \mathrm{~mm}$ from the waist, the polarization was switched to azimuthal and a series of images were recorded when it crossed the waist moving against the light. This routine was repeated three times for each shell to accumulate statistics. The relatively large distance from the return points to the beam waist was necessary to stabilize the shell motion during data recording. Figure $3 c$ presents the results obtained for five different shells.

To measure the wall and Au coating thickness, several representative shells were crushed and then examined under a field-emission scanning electron microscope.

The mass of the shell in Fig. 3d,e was found from Stokes' law by blocking the laser beam and then measuring the terminal velocity of the falling shell.

Received 11 December 2013; accepted 10 September 2014; published online 19 October 2014

\section{References}

1. Lee, S-H., Roichman, Y. \& Grier, D. Optical solenoid beams. Opt. Express 18, 6988-6993 (2010)

2. Ruffner, D. B. \& Grier, D. G. Optical conveyors: a class of active tractor beams. Phys. Rev. Lett. 109, 163903 (2012).

3. Brzobohatý, O. et al. Experimental demonstration of optical transport, sorting and self-arrangement using a 'tractor beam'. Nature Photon. 7, 123-127 (2013)

4. Kajorndejnukul, V., Ding, W., Sukhov, S. \& Dogariu, A. Linear momentum increase and negative optical forces at dielectric interface. Nature Photon. 7, 787-790 (2013).

5. Marston, P. L. Axial radiation force of a Bessel beam on a sphere and direction reversal of the force. J. Acoust. Soc. Am. 120, 3518-3524 (2006).

6. Sukhov, S. \& Dogariu, A. Negative nonconservative forces: optical 'tractor beams' for arbitrary objects. Phys. Rev. Lett. 107, 203602 (2011).

7. Saenz, J. Laser tractor beams. Nature Photon. 5, 514-515 (2011).

8. Chen, J., Ng, J., Lin, Z. \& Chan, C. T. Optical pulling force. Nature Photon. 5, 531-534 (2011).

9. Dogariu, A., Sukhov, S. \& Sáenz, J. J. Optically induced 'negative forces'. Nature Photon. 7, 24-27 (2013)

10. Gahagan, K. T. \& Swartzlander Jr., G. A. Optical vortex trapping of particles. Opt. Lett. 21, 827-829 (1996).

11. Bowman, R. W. \& Padgett, M. J. Optical trapping and binding. Rep. Prog. Phys. 76, 1-28 (2013).

12. Grier, D. G. A revolution in optical manipulation. Nature 424, 810-816 (2003).

13. Ashkin, A. \& Dziedzic, J. M. Optical levitation in high vacuum. Appl. Phys. Lett. 28, 333-335 (1976).

14. Peterman, E. J. G., Gittes, F. \& Schmidt, C. F. Laser-induced heating in optical traps. Biophys. J. 84, 1308-1316 (2003).

15. Preining, O. in Aerosol Science (ed. Davies, C. N.) Ch. V, 111-135 (Academic, 1966).

16. Phuoc, T. X. A comparative study of the photon pressure force, the photophoretic force, and the adhesion von der Waals force. Opt. Commun. 245 27-35 (2005).

17. Rohatschek, H. Semi-empirical model of photophoretic forces for the entire range of pressures. J. Aerosol Sci. 26, 717-734 (1995).

18. Wurm, G. \& Krauss, O. Dust eruptions by photophoresis and solid state greenhouse effects. Phys. Rev. Lett. 96, 134301 (2006).

19. Yalamov, Yu. I., Kutukov, V. B. \& Shchukin, E. R. Theory of the photophoretic motion of the large-size volatile aerosol particle. J. Colloid Interface Sci. 57, 564-571 (1976).

20. Hidy, G. M. \& Brock, J. R. Photophoresis and the descent of particles into the lower stratosphere. J. Geophys. Res. 72, 455-460 (1967).

21. Shvedov, V. G. et al. Giant optical manipulation. Phys. Rev. Lett. 105, 118103 (2010).

22. Mackowski, D. W. Photophoresis of aerosol particles in the free molecular and slip-flow regimes. Int. J. Heat Mass Transfer 32, 843-854 (1989).

23. Zulehner, W. \& Rohatschek, H. Representation and calculation of photophoretic forces and torques. J. Aerosol Sci. 26, 201-210 (1995).

24. Yalamov, Yu. I. \& Khasanov, A. S. Photophoresis of coarse aerosol particles with nonuniform thermal conductivity. Tech. Phys. 43, 347-352 (1998). 
25. Dusel, P. W., Kerker, M. \& Cooke, D. D. Distribution of absorption centers within irradiated spheres. J. Opt. Soc. Am. 69, 55-59 (1979).

26. Esseling, M., Rose, P., Alpmann, C. \& Denz, C. Photophoretic trampolineinteraction of single airborne absorbing droplets with light. Appl. Phys. Lett. 101, 131115 (2012).

27. Shvedov, V. G., Hnatovsky, C., Rode, A. V. \& Krolikowski, W. Robust trapping and manipulation of airborne particles with a bottle beam. Opt. Express 19, 17350-17356 (2011).

28. Shvedov, V. G., Hnatovsky, C., Eckerskorn, N., Rode, A. V. \& Krolikowski, W. Polarization-sensitive photophoresis. Appl. Phys. Lett. 101, 051106 (2012)

29. Soong, C. Y., Li, W. K., Liu, C. H. \& Tzeng, P. Y. Theoretical analysis for photophoresis of a microscale hydrophobic particle in liquids. Opt. Express 18, 2168-2182 (2010).

30. Rakic, A. D., Djurisic, A. B., Elazar, J. M. \& Majewski, M. L. Optical properties of metallic films for vertical-cavity optoelectronics devices. Appl. Opt. 37, 5271-5283 (1998).

\section{Acknowledgements}

The authors acknowledge support from the Australian Research Council.

\section{Author contributions}

V.S. conceived the idea. V.S., C.H. and W.K. designed and conducted the experiments. A.R.D. and N.E. developed the theoretical description. A.R.D. performed the numerical simulations. C.H. and V.S. co-wrote the paper. W.K. supervised the project. All authors contributed to the discussion and data analysis.

\section{Additional information}

Reprints and permissions information is available online at www.nature.com/reprints. Correspondence and requests for materials should be addressed to W.K.

\section{Competing financial interests}

The authors declare no competing financial interests. 